\title{
Retrospektive auf die Prävention und Therapie von Covid-19 mit Chinesischer Medizin in China
}

\author{
von Hui Zhang (Åbyhøj, Dänemark), Lu Luo (Beijing), Zhi Qing Liu (Chengdu), Hui Xie (Chengdu)
}

Zusammenfassung: Die Chinesische Medizin hat im Kampf gegen Covid-19 in China eine wichtige Rolle dabei gespielt, die Symptome zu lindern, eine Weiterentwicklung von der leichten oder üblichen zur schweren Form zu reduzieren, die Genesungsrate zu steigern, die Mortalität zu senken und die Rekonvaleszenz zu fördern. Dieser Artikel geht auf die Krankheitsagenzien und -mechanismen der Krankheit ein und schildert, welche Phasen und Formen differenziert werden. Auf dieser Basis fasst er die Ergebnisse von einigen bereits veröffentlichten chinesischen Studien zusammen, die zur Behandlung von Covid-19 mit Chinesischer Medizin in Kombination mit westlicher Medizin durchgeführt wurden. Die Autoren gehen näher auf die empfohlene Behandlung mit Akupunktur und klassischen Rezepturen ein. Sie beschreiben verschiedene Maßnahmen, die in den staatlichen Behandlungsplänen zur Prävention vorgeschlagen werden. Für die Phase der Rekonvaleszenz werden neben Akupunktur, Moxibustion und Phytotherapie auch chinesische Bewegungstherapien empfohlen.

Schlüsselwörter: Covid-19 - Akupunktur · Chinesische Phytotherapie · Studien · Klassische Rezepturen · Prävention · Rekonvaleszenz

Chin Med 2020;35:129-142

\section{Retrospective on the use of Chinese Medicine for the prevention and therapy of Covid-19 in China}

Abstract: In the fight against Covid-19, Chinese Medicine had an important role to play in alleviating the symptoms, in hindering progression from the mild or common forms of the illness to the more severe form, in increasing the rate of recovery, in reducing mortality and in promoting convalescence. This article discusses the agents and mechanisms of the illness and describes the differentiation made between the various phases and forms. On this basis, it summarizes the results of several previously published Chinese studies that were conducted on the treatment of Covid-19 with Chinese Medicine in combination with Western medicine.

The authors go into detail about the recommended treatment using acupuncture and classical prescriptions. They describe various measures proposed in the state treatment plans for prevention. For the convalescence phase, Chinese movement therapies are recommended in addition to acupuncture, moxibustion and phytotherapy.

Key Words: Covid-19 · Acupuncture · Chinese phytotherapy · Studies · Classical prescriptions · Prevention · Convalescence 
Der Einsatz von Chinesischer Medizin in chinesischen Krankenhäusern

Die Pathologie von Covid-19 aus chinesischer Sicht

\section{1 Überblick über die Behandlung von Covid-19 in China}

Die Chinesische Medizin hat eine lange Tradition der Behandlung von epidemischen Krankheiten, zu denen auch die durch das neuartige Coronavirus verursachte Pneumonie (Covid-19) zu rechnen ist. Von den in China bis zum Zeitpunkt des Verfassens dieses Artikels bestätigten Covid-19-Fällen wurden 74.187 mit Chinesischer Medizin behandelt, das sind 91,5\% aller bestätigten Fälle (Wang Wei 2020). Wie Berichte aus China zeigen, lassen sich mit Chinesischer Medizin die Symptome lindern, die Weiterentwicklung von der leichten und üblichen Form zur schweren Form reduzieren, die Genesungsrate steigern, die Mortalität senken und die Rekonvaleszenz beschleunigen (Luo 2020). In den Krankenhäusern wurden auf der Basis von Standardrezepturen wie „Den o. pulmonalis (Fk ,Lunge') entfaltendes und giftlösendes Dekokt" (Xuanfei baidu tang) ${ }^{1}$ und „Den o. pulmonalis (Fk ,Lunge') kühlendes und Toxisches ausscheidendes Dekokt" (Qingfei paidu tang) ${ }^{2}$ je nach den Symptomen des Patienten individuelle Rezepturen zusammengestellt, wobei je nach Bedarf zusätzlich mit Akupunktur, Moxibustion oder Ohrakupressur etc. behandelt wurde. Auf diese Weise konnten sehr gute Resultate erzielt werden (Shi Suofang 2020).

\section{Erkenntnisse der Chinesischen Medizin zu Covid-19}

\subsection{Krankheitsagenzien und -mechanismen}

Bei Covid-19 dringt eine epidemische Heteropathie (epidemische Schrägläufigkeit, yili 疫戻) über Mund und Nase ein, anschließend breitet sie sich von der extima (Oberfläche, biao) in die intima (Inneres, $l i$ ) aus. Die Heteropathie (Schrägläufigkeit, xie) führt zur Einstauung des qi pulmonale (Qi des Fk „Lunge“, feiqi), und das mittlere Calorium (mittlerer Wärmebereich, zhongjiao) wird bedrängt. Die oo. lienalis et stomachi (Fk „Milz" und „Magen“, pi wei) und der o. intestini crassi (Fk „Dickdarm“, dachang) oder auch der gesamte Körper werden ebenfalls betroffen, wobei das Übergreifen und die Veränderungen rasch ablaufen. Die Folge sind schließlich Zeichen eines kritischen Zustandes (Chen Renshou 2020).

Die Pathologie von Covid-19 lässt sich mit den sechs Begriffen humor („Feuchtigkeit“, shi), calor („Hitze“, re), Toxisches, Stasen, Trübes und depletio (energetische Schwäche, $x u$ ) zusammenfassen, wobei humor („Feuchtigkeit“, shi) die größte Rolle spielt.

\footnotetext{
1 Das „Den o. pulmonalis (Fk ,Lunge') entfaltende und giftlösende Dekokt” (Xuanfei baidu tang 宣肺败毒汤) besteht aus den vier Rezepturen „Dekokt mit Ephedra, Prunus armeniaca, Gypsum und Glycyrrhiza" (Maxing shigan tang, s. Hempen 2006, S. 404), "Dekokt mit Ephedra, Prunus armeniaca, Coix und Glycyrrhiza" (Maxing yigan tang, bestehend aus Ephedrae herba [Mahuang], Armeniacae semen [Xingren], Glycyrrhizae radix [Gancao] und Coicis semen [Yiyiren]), "Phragmites-Dekokt" (Weijing tang, s. Hempen 2006, S. 134) und "Dekokt mit Lepidium und Jujuba zur Zerstreuung des Fk Lunge (o. pulmonalis)" (Tingli dazao xiefei tang, s. Hempen 2006, S. 410), Quelle: http://www.scio.gov.cn/xwfbh/xwbfbh/wqfbh/42311/42768/zy42772/Document/1675829/1675829.htm.

2 Zusammensetzung des „Den o. pulmonalis (Fk ,Lunge') kühlenden und Toxisches ausscheidenden Dekoktes" (Qingfei paidu tang 清肺排毒汤): $9 \mathrm{~g}$ Ephedrae herba (Mahuang), $6 \mathrm{~g}$ in einer Flüssigkeit geröstete Glycyrrhizae radix (Gancao), $9 \mathrm{~g}$ Armeniacae semen (Xingren), 15-30 g unpräpariertes Gypsum fibrosum (Shigao, vorher abgekocht), $9 \mathrm{~g}$ Cinnamomi cassiae ramulus (Guizhi), $9 \mathrm{~g}$ Alismatis rhizoma (Zexie), $9 \mathrm{~g}$ Polyporus (Zhuling), $9 \mathrm{~g}$ Atractylodis macrocephalae rhizoma (Baizhu), $15 \mathrm{~g}$ Poria (Fuling), $16 \mathrm{~g}$ Bupleuri radix (Chaihu), $6 \mathrm{~g}$ Scutellariae radix (Huangqin), $9 \mathrm{~g}$ in Ingwer präparierte Pinelliae rhizoma (Jiangbanxia), $9 \mathrm{~g}$ Zingiberis rhizoma recens (Shengjiang), $9 \mathrm{~g}$ Asteris radix (Ziwan), $9 \mathrm{~g}$ Farfarae flos (Kuandonghua), $9 \mathrm{~g}$ Belamcandae rhizoma (Shegan), $6 \mathrm{~g}$ Asari radix (Xixin), $12 \mathrm{~g}$ Dioscoreae rhizoma (Shanyao), $6 \mathrm{~g}$ Aurantii fructus immaturus (Zhishi), $6 \mathrm{~g}$ Citri reticulatae pericarpium (Chenpi), $9 \mathrm{~g}$ Agastachis herba (Huoxiang), Quelle: Wang Raoqiong 2020.
} 
Zu Beginn der Affektion dringt bei Covid-19 die Heteropathie (Schrägläufigkeit, xie) noch nicht tief ein, so dass zunächst leichte Symptome des Bereichs des $q i$ defensivum (Wehrenergie, weiqi) wie Fieber, trockener Husten und Kraftlosigkeit im Vordergrund stehen. Da jedoch epidemisches ( $l i$ 戻) Qi zur Erkrankung führt, das sich rasch weiterentwickelt, kann es schnell den Bereich des Qi passieren und in den Bereich des qi constructivum (Bauenergie, yingqi) eindringen. Ardor („Glut“, huo) wirkt kontravektiv auf den o. pericardialis (Fk „Herzbeutel“, xinbao), und in der Tiefe werden die Funktionskreise affiziert. Zudem ist humor („Feuchtigkeit“, shi) vom Wesen her schwer, so dass der Krankheitsverlauf lang ist und das orthopathische Qi (geradläufiges Qi, zhengqi) geschmälert wird. Die Analyse aus einer kombiniert chinesisch-westlichen Sicht ergibt, dass Einstauungen von humor („Feuchtigkeit“, shi), Toxischem und epidemischem Qi sich im Inneren in den Funktionskreisen zu calor („Hitze“, re) wandeln, es werden Stasen hervorgebracht, das qi constructivum (Bauenergie, yingqi) und das Xue werden verbrannt, und sie verbreiten sich in den drei Calorien (drei Wärmebereiche, sanjiao). Es werden zahlreiche kritische Symptome wie Acute Respiratory Distress Syndrome (ARDS) und Sepsis hervorgerufen, was zum Tod des Patienten führen kann (Peng Bo 2020).

\subsection{Therapie}

\subsubsection{Festlegung der Behandlung entsprechend den vier Phasen}

In den Behandlungsplänen, die landesweit und in den einzelnen Provinzen veröffentlicht wurden und weitgehend einheitlich sind, werden für die Chinesische Medizin in der klinischen Praxis die Phasen Anfangsphase, mittlere Phase, Phase mit schweren Symptomen und Rekonvaleszenzphase differenziert.

Huang Xiaoqing et al. (Huang Xiaoqing 2020) haben in der Provinz Guangdong 35 Covid-19-Patienten untersucht, von denen bei $80 \%$ Veränderungen im LungenCT feststellbar waren. Bei 45,71 \% der Patienten lagen Einstauungen von heteropathischem humor (schrägläufige „Feuchtigkeit“, shixie) im o. pulmonalis (Fk "Lunge“, fei) vor und bei 37,14 \% Einstauungen von heteropathischem calor (schrägläufige „Hitze“, shire) im o. pulmonalis (Fk „Lunge“, fei). Die größte Rolle spielt also humor („Feuchtigkeit“, shi), gefolgt von calor („Hitze“, re). Der Zungenbelag war zumeist klebrig bei einem roten oder blassroten Zungenkörper. Zhou Jing et al. (Zhou Jing 2020) haben in der Provinz Anhui bei 144 Patienten eine Analyse aus Sicht der Chinesischen Medizin durchgeführt und festgestellt, dass das Auftreten der Erkrankung in einem engen Zusammenhang mit den oo. pulmonalis et lienalis (Fk „Lunge“ und "Milz“, fei pi) stand, wobei die Heteropathie (Schrägläufigkeit, xie) Epidemisch-Toxisches (yidu) zusammen mit humor („Feuchtigkeit“, shi) den wichtigsten pathologischen Faktor darstellte. Pan Xiaoqiong et al. (Pan Xiaoqiong 2020) haben bei einer klinischen Beobachtung festgestellt, dass die wichtigste Symptomkonfiguration bei der üblichen Form von Covid-19 Einlagerungen von humor („Feuchtigkeit“, shi), calor („Hitze“, re) und Epidemisch-Toxischem (yidu) im o. pulmonalis (Fk „Lunge“, fei) war. Die involvierten pathologischen Faktoren waren algor („Kälte“, han), calor („Hitze“, re), humor („Feuchtigkeit“, shi), Toxisches $(d u)$ und depletio (energetische Schwäche, $x u$ ), wobei humor-Toxisches („Feuchtigkeit“-Toxisches, shidu) den zentralen Krankheitsmechanismus bildete.

Bei der Behandlung sollte humor („Feuchtigkeit“, shi) im Mittelpunkt stehen, wobei vor allem bei der leichten und bei der üblichen Form calor („Hitze“, re)
Differenzierung der einzelnen Phasen und Formen 
Die wichtigsten Therapieprinzipien

Der Einsatz von „schwebender Moxibustion" kühlende und den o. pulmonalis (Fk „Lunge“, fei) entfaltende, Toxisches $(d u)$ herauslösende und „Schmutziges“ (hui) beseitigende, den o. lienalis (Fk „Milz“, pi) kräftigende und humor („Feuchtigkeit“, shi) eliminierende, ventus („Wind“, feng) lösende und die reticulares (Netzleitbahnen, luomai) durchgängig machende Methoden eingesetzt werden sollten, um zu verhindern, dass es zu einer Weiterentwicklung der Erkrankung kommt. (Peng Bo 2020)

\subsubsection{Akupunkturbehandlung}

Obwohl bei der Behandlung von Covid-19 mit Chinesischer Medizin die chinesischen Arzneimittel die führende Rolle spielen, werden seit alters her auch Akupunktur und Moxibustion in großem Umfang zur Prävention und Therapie von „Epidemischen Wärme-Erkrankungen“ (wenyi 瘟疫) genutzt. Zur Intervention mit Akupunktur und Moxibustion bei akuten Infektionskrankheiten der Gegenwart wie epidemisches hämorrhagisches Fieber, Influenza, akute bakterielle Dysenterie, Meningitis cerebrospinalis epidemica, Virushepatitis, Hand-FußMund-Krankheit bei Kindern, SARS etc. existieren jeweils klare, verlässliche Berichte zur Therapiewirkung (Liu Bing 2020). Der Chinesische Verband für Akupunktur und Moxibustion hat den „Leitplan für die Intervention mit Akupunktur und Moxibustion bei Covid-19 (2. Auflage)“ ausgearbeitet (Shi Xuemin 2020), wobei bei der Aku-Moxi-Therapie streng auf Abstand und Desinfektion zu achten ist.

Huang Xianbao et al. (Huang Xianbao 2020) haben bei 42 Covid-19-Patienten (übliche Form) im Bereich der Foramina Rs8/Ren8 („Mitte des Nabels“, shenque) und S25/Ma25 („Angel des Himmels“, tianshu) „schwebende Moxibustion“ $(\text { reminjiu })^{3}$ durchgeführt. Dabei zeigte sich, dass „schwebende Moxibustion“ (reminjiu) bei Covid-19-Patienten wirksam negative Emotionen reduzieren und Druckgefühle im Thorax und Appetitlosigkeit bessern kann und sie von den Patienten gut angenommen wird.

Die Akupunkturintervention wird je nach Entwicklung des Krankheitsmechanismus entsprechend den drei Phasen „medizinische Beobachtungsphase“,

Tabelle 1: Behandlungsplan Akupunktur bei Covid-19

\begin{tabular}{|c|c|c|c|}
\hline Phase & Therapieziel & Wichtigste Foramina & Ergänzende Foramina \\
\hline \multirow[t]{3}{*}{$\begin{array}{l}\text { Medizinische } \\
\text { Beobachtungs- } \\
\text { phase (Ver- } \\
\text { dachtsfälle) }\end{array}$} & \multirow{3}{*}{$\begin{array}{l}\text { Stimulieren des orthopa- } \\
\text { thischen Qi (geradläufiges } \\
\text { Qi, zhengqi) und der Funkti- } \\
\text { onen der oo. pulmonalis et } \\
\text { lienalis (Fk „Lunge“ und } \\
\text { "Milz", fei pi), Eliminieren } \\
\text { der epidemischen Hetero- } \\
\text { pathie („Schrägläufigkeit”, } \\
\text { xie), so dass die Funktions- } \\
\text { kreise Heteropathien } \\
\text { (,Schrägläufigkeiten“, xie) } \\
\text { besser abwehren können }\end{array}$} & \multirow{2}{*}{$\begin{array}{l}\text { (1)V12/BI12 („Pforte der Winde“, fengmen), } \\
\text { V13/BI13 („Einflusspunkt des,Lungen'- } \\
\text { Funktionskreises", feishu), V20/BI20 („Ein- } \\
\text { flusspunkt des,Mitten'-Funktionskreises”, } \\
\text { pishu); } \\
\text { (2) IC4/Di4 („Vereinte Täler“, hegu), } \\
\text { IC11/Di11 („Gekrümmter Teich“, quchi), } \\
\text { P5/Lu5 („Moorsee am Fußpunkt“, chize), } \\
\text { P10/Lu10 („Fischbauchgrenze“, yuji); }\end{array}$} & $\begin{array}{l}\text { Fieber, trockener Rachen, trockener Husten: zusätzlich } \\
\text { Rg14/Du14 („Punkt aller Strapazen“, dazhui), Rs22/Ren22 } \\
\text { („Bresche des Himmels“, tiantu), P6/Lu6 („Die Tiefe des } \\
\text { Lochs“, kongzui) }\end{array}$ \\
\hline & & & $\begin{array}{l}\text { Übelkeit, halbflüssiger Stuhl, vergrößerter Zungenkörper } \\
\text { mit klebrigem Belag, pp. lenes („nachgiebige“ Pulse, } \\
\text { rumai): Rs12/Ren12 („Sammlungspunkt des,Magen'-Funk- } \\
\text { tionskreises“, zhongwan), S25/Ma25 („Angel des Himmels“, } \\
\text { tianshu), S40/Ma40 („Üppige Fülle“, fenglong) }\end{array}$ \\
\hline & & $\begin{array}{l}\text { (3) Rs6/Ren6 („Meer des Qi“, qihai), } \\
\text { S36/Ma36 („Dritter Weiler am Fuß", zusanli), } \\
\text { L6/Mi6 („Die Verbindung der drei Yin“, } \\
\text { sanyinjiao); }\end{array}$ & $\begin{array}{l}\text { Gleichzeitig Abgeschlagenheit, Kraftlosigkeit, Appetitlo- } \\
\text { sigkeit: Rs12/Ren12 (,Sammlungspunkt des,Magen'-Funk- } \\
\text { tionskreises“, zhongwan), vier periumbilikale Foramina } \\
\text { (jeweils } 1 \text { cun über, unter, links und rechts von der Nabel- } \\
\text { mitte), V20/BI20 („Einflusspunkt des,Mitten'-Funktions- } \\
\text { kreises“, pishu) }\end{array}$ \\
\hline
\end{tabular}

3 Bei der "schwebenden Moxibustion“ (reminjiu 热敏炎) wird die brennende Moxazigarre in einem bestimmten Abstand über dem Foramen gehalten. (Anm. d. Übers.) 
Tabelle 1: Behandlungsplan Akupunktur bei Covid-19

Klinische Anregen des orthopathi- (1) IC4/Di4 („Vereinte Täler“, hegu), H3/Le3 Behandlungs- $\quad$ schen Qi (geradläufiges Qi, („Die mächtige große Straße“, taichong), phase (bestä- zhengqi) der oo. pulmonalis Rs22/Ren22 („Bresche des Himmels", tigte Diagnose) et lienalis ( $\mathrm{Fk}_{\text {„Lunge" und }}$ „Milz", fei pi), Schützen der Funktionskreise und Reduzieren der Läsionen, Vertreiben der epidemischen Heteropathie (,Schrägläufigkeit", xie), Hervorbringen der Wandlungsphase Metall durch Stützen der Wandlungsphase Erde, Unterbrechen des Krankheitszustandes, Lösen der Emotionen, Stärken des Vertrauens in das Besiegen der Heteropathie (,Schrägläufigkeit", xie) ,Lungen'-Funktionskreises", feishu), zhongfu), Rs17/Ren17 („Vorhof der Brust", shanzhong), Rs6/Ren6 ("Meer des Qi", tiantu), P5/Lu5 („Moorsee am Fußpunkt", chize), P6/Lu6 („Die Tiefe des Lochs", kongzui), S36/Ma36 („Dritter Weiler am Fuß”, zusanli), L6/Mi6 („Die Verbindung der drei Yin", sanyinjiao);

(2) V11/BI11 ("Großes Weberschiffchen", dazhu), V12/BI12 („Pforte der Winde“, fengmen), V13/BI13 („Einflusspunkt des V15/BI15 („Einflusspunkt des,Herz'Funktionskreises", xinshu), V17/BI17 („Einflusspunkt des Zwerchfells", geshu);
Nicht weichendes Fieber: zusätzlich Rg14/Du14 („Punkt aller Strapazen", dazhui), IC11/Di1 1 ("Gekrümmter Teich", quchi); oder blutiges Nadeln von Ex15/Ex-UE-11 („Die zehn Drainagen“, shixuan) und F8/Gb8 („Der obere Punkt des Ohres", erjian)

Druckgefühle im Thorax und Kurzatmigkeit: Pc6/Pe6 (,Inneres Passtor", neiguan), P7/Lu7 („Reihe von Lücken“, lieque); oder Rs14/Ren14 („Sammlungspunkt des,Herz'Funktionskreises“, juque), H14/Le14 („Zusammenkunftspunkt des,Leber'-Kreises", qimen), R6/Ni6 („Das Meer der Erhellung", zhaohai)

Husten mit Auswurf: zusätzlich P7/Lu7 (,Reihe von Lücken“, lieque), S40/Ma40 („Üppige Fülle“, fenglong), Ex10/Ex-B-1 („Asthmaerleichterung“, dingchuan)

(3) P1/Lu1 ("Versammlungshalle der Mitte“, qihai), Rs4/Ren4 („Das erste der Passtore“, guanyuan), Rs12/Ren 12 („Sammlungspunkt des,Magen'-Funktionskreises", zhongwan);

bei leichter und üblicher Form jedes Mal aus den Foramina der Gruppen (1) und (2) 2-3 Foramina auswählen; bei schwerer Form aus den Foramina der Gruppe (3) 2-3 Foramina auswählen

Diarrhoe mit halbflüssigem Stuhl: zusätzlich S25/Ma25 („Angel des Himmels", tianshu), S37/Ma37 („Obere Enge des weiten Feldes", shangjuxu)

Auswurf von gelblichem, klebrigem Sputum, Obstipation: zusätzlich Rs22/Ren22 („Bresche des Himmels", tiantu), T6/3E6 („Der fliegende Tiger", feihu, bzw., Seitlicher Abzugsgraben", zhigou), S25/Ma25 ("Angel des Himmels", tianshu), S40/Ma40 („Üppige Fülle", fenglong)

Subfebrile Temperaturen, eventuell keine Temperaturerhöhung, Übelkeit und Erbrechen, halbflüssiger Stuhl, blasser oder blassroter Zungenkörper, weißer oder weißer, klebriger Belag: zusätzlich V13/BI13 („Einflusspunkt des,Lungen'-Funktionskreises", feishu), S25/Ma25 („Angel des Himmels", tianshu), L14/Mi14 („Knoten im Abdomen“, fujie), Pc6/Pe6 („Inneres Passtor", neiguan)

Deutliche Symptome der "Verbindungen des o. pulmona-

Rekonvaleszenz- Eliminieren von residualem Pc6/Pe6 („Inneres Passtor“, neiguan), phase Toxischen, WiederherstelS36/Ma36 ("Dritter Weiler am Fuß“, zusar Rs12/Ren12 ("Sammlungspunkt des len des qi primum (ursprüngliches Qi, yuanqi), Fördern der Regeneration der Funktionskreise, Wiederherstellen der FunktioS25/Ma25 („Angel des Himmels", tianshu), Rs6/Ren6 („Meer des Qi“, qihai) nen der oo. pulmonalis et

lienalis ( $\mathrm{Fk}_{\text {"Lunge" }}$ und

„Milz", fei pi) lis" (feixi) wie Kurzatmigkeit: zusätzlich Rs17/Ren17 („Vorhof der Brust", shanzhong), V13/B113 („Einflusspunkt des,Lungen'-Funktionskreises", feishu), P1/Lu1

(,Versammlungshalle der Mitte", zhongfu)

Deutliche Symptome der oo. lienalis et stomachi ( $\mathrm{Fk}_{\text {"Milz" }}$ und "Magen", pi wei) wie Appetitlosigkeit, Diarrhoe: zusätzlich Rs13/Ren13 („Ort des Mageneingangs“, shangwan), L9/Mi9 („Die Quelle am Yin-Grabhügel“, yinlingquan)

Starke Mundtrockenheit, starker Durst: zusätzlich R3/Ni3 („Mächtiger Wasserlauf", taixi), T4/3E4 („Teich des Yang“, yangchi)

Deutliche Palpitationen: zusätzlich V15/BI15 („Einflusspunkt des,Herz'-Funktionskreises", xinshu), V14/BI14 („Einflusspunkt für das, weichende Yin'", jueyinshu);

starkes Schwitzen: zusätzlich IC4/Di4 (,Vereinte Täler“, hegu), R7/Ni7 („Der zurückfließende Strom", fuliu), S36/Ma36 („Dritter Weiler am Fuß", zusanli)

Schlafstörungen: zusätzlich C7/He7 („Pforte des Shen“, shenmen), Ex1/Ex-HN-3 (,Siegelhalle", yintang),

Ex5/Ex-HN-54 („Ruhiger Schlaf“, anmian), R1/Ni1 („Die emporsprudelnde Quelle“, yongquan) 
„klinische Behandlungsphase“ und „Rekonvaleszenzphase“ durchgeführt. Dabei werden die Symptomkonfigurationen nach den Funktionskreisen und Leitbahnen bestimmt, und dementsprechend werden die Hauptforamina ausgewählt. Diese werden je nach den klinischen Symptomen modifiziert, wobei man sich an dem Grundsatz „Auswahl von wenigen, aber guten Punkte“ orientieren sollte. Tabelle 1 fasst den wesentlichen Inhalt des „Leitplans für die Intervention mit Akupunktur und Moxibustion bei Covid-19 (2. Auflage)“ zusammen.

\subsubsection{Einsatz von klassischen Rezepturen}

Xu Erping (Xu Erping 2020) hat auf Basis der „Abhandlung über schädigende ,Kälte (algor laedens) und verschiedene Erkrankungen (morbi varii)“ (Shanghan zabing lun, von Zhang Zhongjing Anfang des 3. Jh. verfasst) empfehlenswerte klassische Rezepturen für die Behandlung von Covid-19 zusammengestellt, s. Tabelle 2.

Tabelle 2: Der Einsatz von klassischen Rezepturen bei der Behandlung von Covid-19

\begin{tabular}{|c|c|c|}
\hline $\begin{array}{l}\text { Wichtigstes } \\
\text { Symptom }\end{array}$ & Form & Klassische Rezeptur \\
\hline \multirow[t]{3}{*}{ Fieber } & $\begin{array}{l}\text { Einstauungen von humor algidus } \\
\text { (,Kälte-Feuchtigkeit“, hanshi) im } \\
\text { o. pulmonalis (Fk "Lunge“, fei) }\end{array}$ & „Pueraria-Dekokt mit Pinellia“ (Gegen jia banxia tang, s. Scheid 2009, S. 21) \\
\hline & $\begin{array}{l}\text { In den o. pulmonalis (Fk „Lunge", fei) } \\
\text { eindringender calor humidus } \\
\text { („Feuchtigkeit-Hitze“, shire) }\end{array}$ & $\begin{array}{l}\text { „Kleines Bupleurum-Dekokt“" (Xiao chaihu tang, s. Hempen 2006, S. 156) mit,"Dekokt mit } \\
\text { Ephedra, Prunus armeniaca, Coix und Glycyrrhiza“ (Maxing yigan tang, s.o.) }\end{array}$ \\
\hline & $\begin{array}{l}\text { Blockade des o. pulmonalis (Fk "Lunge", } \\
\text { fei) durch Epidemisch-Toxisches }\end{array}$ & $\begin{array}{l}\text { "Dekokt mit Ephedra, Prunus armeniaca, Gypsum und Glycyrrhiza" (Maxing shigan tang, s. Hem- } \\
\text { pen 2006, S. 404) und "Großes Bupleurum-Dekokt" (Da chaihu tang, s. Hempen 2006, S. 158) mit } \\
\text { "Dekokt des Weißen Tigers mit Ginseng" (Baihu jia renshen tang, s. Scheid 2009, S. 154) }\end{array}$ \\
\hline \multirow[t]{2}{*}{ Sputum } & $\begin{array}{l}\text { Einstauung von calor pituitae } \\
\text { ('Schleim-Hitze“, tanre) im } \\
\text { o. pulmonalis (Fk, "Lunge“, fei) }\end{array}$ & $\begin{array}{l}\text { „Das Dekokt der Magd aus Yue“ (Yuebi tang, s. Scheid 2009, S. 185) mit „Kleines Dekokt } \\
\text { gegen Vernichtungsgefühl in der Brust" (Xiao xianxiong tang, s. Hempen 2006, S. 252) mit } \\
\text { „Phragmites-Dekokt" (Weijing tang, s. Hempen 2006, S. 134) }\end{array}$ \\
\hline & $\begin{array}{l}\text { Blockade des o. pulmonalis (Fk "Lunge", } \\
\text { fei) durch humor pituitae } \\
\text { (,Schleim-Feuchtigkeit", tanshi) }\end{array}$ & $\begin{array}{l}\text { „Kleines Dekokt des grünen Drachen“ (Xiao qinglong tang, S. Hempen 2006, S. 48) mit } \\
\text { "Pulver der fünf Ling-Bestandteile" (Wuling san, s. Hempen 2006, S. 208) }\end{array}$ \\
\hline \multirow[t]{2}{*}{ Kurzatmigkeit } & $\begin{array}{l}\text { Repletive Kurzatmigkeit (algor-",Wasser" } \\
\text { ["Kälte-Wasser", hanshui] beeinträchtigt } \\
\text { den o. pulmonalis [Fk "Lunge“, fei]) }\end{array}$ & $\begin{array}{l}\text { "Dekokt mit Belamcanda und Ephedra" (Shegan mahuang tang, s. Hempen 2006, S. 414) mit } \\
\text { "Dekokt mit Poria, Cinnamomum, Atractylodes und Glycyrrhiza" (Linggui zhugan tang, } \\
\text { s. Hempen 2006, S. 210) }\end{array}$ \\
\hline & $\begin{array}{l}\text { Depletive Kurzatmigkeit (depletio } \\
\text { [energetische Schwäche, xu] des } \\
\text { qi pulmonale et renale [Qi der Fk } \\
\text { "Lunge" und „Niere", fei shen qi]) }\end{array}$ & $\begin{array}{l}\text { "Aconit-Dekokt" (Fuzi tang, s. s. Scheid 2009, S. 747) mit „Dekokt mit Stephania und } \\
\text { Astragalus" (Fangji huangqi tang, s. Hempen 2006, S. 216) mit „Dekokt mit Zimtzweigen, } \\
\text { Süßholz, Mastodi fossilium und Ostrea" (Guizhi gancao longgu muli tang, s. Scheid 2009, } \\
\text { S. 365) }\end{array}$ \\
\hline \multirow[t]{2}{*}{ Obstipation } & Repletive Obstipation & "Großes Dekokt zur Wiederherstellung des Qi-Flusses" (Da chengqi tang, s. Hempen 2006, S. 470) \\
\hline & Depletive Obstipation & $\begin{array}{l}\text { "Cannabis-Pille" (Maziren wan, s. Hempen 2006, S. 484) mit „Dekokt mit Lilium und } \\
\text { Rehmannia" (Baihe dihuang tang, s. Scheid 2009, S. 220) und „Pille aus unreifen Zitronen und } \\
\text { Atractylodes" (Zhizhu wan, s. Scheid 2009, S. 837) }\end{array}$ \\
\hline \multirow{2}{*}{$\begin{array}{l}\text { Benommenheit } \\
\text { und Bewusst- } \\
\text { losigkeit }\end{array}$} & Yang-Blockade & $\begin{array}{l}\text { „Dekokt des weißen Tigers" (Baihu tang, s. Hempen 2006, S. 100) mit „Großes Dekokt zur } \\
\text { Wiederherstellung des Qi-Flusses" (Da chengqi tang, s. Hempen 2006, S. 470) }\end{array}$ \\
\hline & Yin-Blockade & $\begin{array}{l}\text { "Dekokt aus den frischen Trieben des Aconits" (Wutou tang, s. Scheid, S. 624) mit „Dekokt gegen } \\
\text { die vier Gegenläufigkeiten mit Angelica sinensis" (Danggui sini tang, s. Hempen 2006, S. 80) }\end{array}$ \\
\hline $\begin{array}{l}\text { "Entweichen- } \\
\text { Symptomatik" } \\
\text { (tuozheng) }\end{array}$ & Verlust und Entweichen des Yang-Qi & $\begin{array}{l}\text { „Das Dekokt gegen die vier Kontravektionen“ (Sini tang, s. Scheid, S. 274) mit Ginseng radix } \\
\text { (Renshen) }\end{array}$ \\
\hline
\end{tabular}




\section{Prävention}

Die staatlichen Gesundheitsbehörden und viele Krankenhäuser haben sehr früh Pläne zur Prävention von Covid-19 entwickelt. Die wichtigsten Rezepturen sind dabei das „Pulver gegen Wind aus Jade“ (Yu pingfeng san, s. Hempen 2006, S. 342) und das „Dekokt mit Cinnamomum“ (Guizhi tang, s. Hempen 2006, S. 392), oder es werden das „Pulver gegen Wind aus Jade“ (Yu pingfeng san, s. Hempen 2006, S. 342, als Pille), die „Die Orthopathie stützende Pille mit Codonopsis und Astragalus" (Shenqi fuzheng wan, als Kapsel) ${ }^{4}$ oder die "Tablette mit Codonopsis und Astragalus“ (Shenqi pian, als Kapsel) ${ }^{5}$ eingesetzt. Für Zuhause gibt es zahlreiche Empfehlungen:

(a) Aromatherapie: Räuchern mit Moxazigarren oder chinesischen Arzneimitteln - nur mit Atractylodis rhizoma (Cangzhu) oder mit mehreren chinesischen Arzneimitteln, ausgewählt aus Isatidis radix (Banlangen), Acori rhizoma (Shichangpu), Dryopteridis rhizoma (Guanzhong) und Lonicerae flos (Jinyinhua);

(b) Mitführen von Duftbeuteln mit chinesischen Arzneimitteln: $10 \mathrm{~g}$ Atractylodis rhizoma (Cangzhu), $10 \mathrm{~g}$ Artemisiae argyi folium (Aiye), $10 \mathrm{~g}$ Acori rhizoma (Shichangpu), $10 \mathrm{~g}$ Menthae herba (Bohe), $10 \mathrm{~g}$ Agastachis herba (Huoxiang);

(c) Diätetik: Empfehlenswerte Lebensmittel zum Kühlen von calor („Hitze“, re) sind Spargel, Löwenzahn, Houttuynia-Kraut und Lotos-Samen. Zum Regulieren des qi pulmonale (Qi des Fk „Lunge“, feiqi) und Beseitigen von pituita („Schleim“, tan) eignen sich Rettich und Hiobstränensamen. Zum Stützen von qi et yin pulmonale (Qi und Yin des Fk „Lunge“, fei qi yin) können Silbermorchel, Lilienzwiebel und Yamswurzel eingesetzt werden (Chen Peiyi 2014).

(d) Bewegung: Es sollten langsame Bewegungsarten wie Taijiquan, „Acht-BrokatÜbungen“ (Baduan jin), „Spiel der Fünf Tiere“ (Wuqinxi) etc. gewählt werden, bei denen man nur leicht ins Schwitzen kommt (s.u.).

Wie die Erfahrungen mit SARS und MERS gezeigt haben, entwickelt ein Teil der Patienten während und nach der Erkrankung Stresszustände wie Depressionen und Angststörungen, wobei vor allem bei schwer erkrankten Patienten die Häufigkeit einer Posttraumatischen Belastungsstörung (PTBS) stark ansteigt. Daher ist während und nach einer Covid-19-Erkrankung eine frühzeitige psychologische Intervention (Li Yuefeng 2020) sinnvoll.
Empfohlene vorbeugende Rezepturen

Zuhause anwendbare präventive Maßnahmen

\footnotetext{
4 Die Ingredienzen der "Die Orthopathie stützenden Pille mit Codonopsis und Astragalus” (Shenqi fuzheng wan 参芪扶正丸) sind Ginseng radix (Renshen), Astragali radix (Huangqi), Hedyotidis diffusae herba (Baihuasheshecao), Scutellariae herba (Banzhilian), Cremastrae pseudobulbus (Shancigu), Curcumae zedoariae rhizoma (Ezhu), Notoginseng radix (Sanqi), Carthami flos (Honghua), Coicis semen (Yiyiren), Poria (Fuling), Eleutherococci senticosi radix et caulis (Ciwujia), Atractylodis macrocephalae rhizoma (Baizhu), Ligustri lucidi fructus (Nüzhenzi) und Lycii fructus (Gouqizi). Sie wirkt das Qi suppletierend, das Xue dynamisierend und Toxisches herauslösend; in der klinischen Praxis wird sie zur unterstützenden Behandlung von Lungen- und Magenkarzinomen eingesetzt, bei denen klinisch depletio qi (energetische Schwäche des Qi, qixu) und Xue-Stasen im Vordergrund stehen.

5 Die Ingredienzen der "Tablette mit Codonopsis und Astragalus" (Shenqi pian 参茂片) sind Codonopsis radix (Dangshen) und Astragali radix (Huangqi). Sie wirkt das qi primum (ursprüngliches Qi, yuanqi) suppletierend, und sie kommt bei depletio qi (energetische Schwäche des Qi, qixu) mit geschwächter Konstitution und Kraftlosigkeit der Extremitäten zum Einsatz.
} 
Kombination mehrerer Rehabilitationstechniken

Wahl zwischen Phytotherapie und Akupunktur

\section{Rekonvaleszenz}

Die Behandlungspläne der staatlichen Gesundheitsbehörden und der Krankenhäuser für Covid-19 enthalten auch Empfehlungen für die Rekonvaleszenzphase. Je nach den klinischen Formen und Symptomkonfigurationen werden zielgerichtet Methoden der Chinesischen und der westlichen Medizin kombiniert eingesetzt, so dass durch Rehabilitationstechniken wie Haltungstherapie, Atemübungen, Bewegungstherapien etc. die Atem- und Körperfunktionen des Patienten verbessert werden. Mit Hilfe von Behandlungsmethoden der Chinesischen Medizin wie Tuina, indirekte Moxibustion etc. werden der Qi-Mechanismus durchgängig gemacht und die Funktionen der Funktionskreise wieder hergestellt. Traditionelle Techniken wie Taijiquan, „Acht-Brokat-Übungen“ (Baduan jin) etc. erhöhen die körperliche Leistungsfähigkeit des Patienten und verbessern seine psychische Verfassung. Auf diese Weise konnten sehr gute Behandlungsergebnisse erzielt und wertvolle Erfahrungen gesammelt werden (Xia Wenguang 2020).

In der Rekonvaleszenzphase stellen nicht beseitigte, residuale Heteropathien (Schrägläufigkeiten, xie) und eine depletio (energetische Schwäche, $x u$ ) des orthopathischen Qi (geradläufiges Qi, zhengqi) den grundlegenden Pathomechanismus dar. Den Ort der Erkrankung bildet der o. pulmonalis (Fk „Lunge“, fei), wobei es sich um eine Erkrankung des Bereichs des qi constructivum (Bauenergie, yingqi) und des Bereichs des Qi innerhalb der morbi temperati („Wärme“-Erkrankungen, wenbing) handelt. Während des gesamten Krankheitsverlaufs, besonders aber bei einem protrahierten Verlauf sind chinesische Arzneimittel von entscheidender Bedeutung, denn mit ihnen lassen sich bleibende fibrotische Veränderungen der Lunge weitestgehend vermeiden, was wichtig ist, damit die Lebensqualität des Patienten weiterhin hoch bleibt.

Zhang Yuan et al. (Zhang Yuan 2020) vertreten die Ansicht, dass die Behandlung in der Rekonvaleszenz von Covid-19 vor allem bei depletio (energetische Schwäche, $x u$ ) der oo. pulmonalis et renalis (Fk „Lunge“ und „Niere“, fei shen) und bei depletio qi (energetische Schwäche des Qi, qixu) und Xue-Stasen ansetzen sollte. Im ersten Fall seien die „Abkochung der sechs Edlen für die Wandlungsphasen Metall und Wasser“ (Jinshui liujun jian, s. Scheid 2009, S. 779) und das „Pulver mit Ginseng und Gecko ${ }^{6 “}$ (Renshen gejie san, s. Hempen 2006, S. 530) zu verabreichen, um die oo. pulmonalis et renalis (Fk „Lunge“ und „Niere“, fei shen) zu suppletieren; oder es sollten Foramina wie R3/Ni3 („Mächtiger Wasserlauf“, taixi), V13/Bl13 („Einflusspunkt des ,Lungen'-Funktionskreises“, feishu), V23/Bl23 („Einflusspunkt des ,Nieren'-Funktionskreises“, shenshu), V43/Bl43 („Das Innere“, gaohuang), Ex10/Ex-B-1 („Asthmaerleichterung“, dingchuan) etc. behandelt werden. Im zweiten Fall sollten das „Den Ursprung bewahrende Dekokt" (Baoyuan tang) ${ }^{7}$ und das „Dekokt der vier Bestandteile mit Persicae semen“" (Taoren siwu tang) ${ }^{8}$ verabreicht werden; oder es sollten Foramina wie V17/

\footnotetext{
6 Gecko (Gejie): CITES Appendix II, der Handel mit Wildentnahmen ist nur erlaubt, wenn eine Ausfuhrgenehmigung die Nachhaltigkeit bescheinigt (s. ersten Artikel in diesem Heft).

7 Die Ingredienzen des "Den Ursprung bewahrenden Dekoktes" (Baoyuan tang 保元汤) sind Ginseng radix (Renshen), Astragali radix (Huangqi), Glycyrrhizae radix (Gancao) und Cinnamomi cassiae cortex (Rougui). Es ist indiziert pockenartigen Pusteln mit eingesunkener Spitze bei depletio qi (energetische Schwäche des Qi, qixu).

8 Die Ingredienzen des "Dekoktes der vier Bestandteile mit Persicae semen“ (Taoren siwu tang 桃仁四物汤) sind Angelicae sinensis radicis cauda (Dangguiwei), Chuanxiong rhizoma (Chuanxiong), Paeoniae radix rubra (Chishaoyao), Moutan cortex (Mudanpi), Cyperi rhizoma (Xiangfu) und Corydalidis rhizoma (Yanhusuo), Quelle: https://baike.baidu.com/item/\%E6\%A1\% 83\%E4\%BB\%81\%E5\%9B\%9B\%E7\%89\%A9\%E6\%B1\%A4.
} 
Bl17 („Einflusspunkt des Zwerchfells“, geshu), P6/Lu6 („Die Tiefe des Lochs“, kongzui), IC4/Di4 („Vereinte Täler“, hegu), IC11/Di11 („Gekrümmter Teich“, quchi), L6/Mi6 („Die Verbindung der drei Yin“, sanyinjiao) etc. behandelt werden.

Die traditionellen Bewegungstechniken spielen bei der Wiederherstellung der Lungenfunktion eine wichtige Rolle. Untersuchungen konnten zeigen, dass die traditionellen chinesischen Bewegungstechniken bei Erkrankungen der "Verbindungen des o. pulmonalis“(feixi) wie chronisch obstruktive Lungenerkrankung (COPD), Asthma bronchiale und Cor pulmonale die Lungenfunktion verbessern, Angst lösen und Schlafstörungen reduzieren können, wodurch die Lebensqualität gesteigert wird. Bei den „Acht-Brokat-Übungen“ (Baduan jin) handelt es sich um moderate Bewegungen, die zudem die Muskelkraft und das Thoraxvolumen steigern können (Zhang Hui 2020).

Im „Spiel der Fünf Tiere“ (Wuqinxi) dominiert das „Spiel des Kranichs“ den o. pulmonalis (Fk „Lunge“, fei), es kann den Brustbereich freimachen, das Qi regulieren, den Qi-Mechanismus durchgängig machen und den Gleichgewichtssinn und die Geschmeidigkeit des Körpers erhöhen. Das Ausdehnen und Zusammenziehen im „Spiel des Tigers“ und das Schütteln des Rumpfes im „Spiel des Bären“ steigern durch Bewegen der Extremitäten und des Lendenbereiches die Aktivität des Brustkorbs, sie trainieren die Funktionen der Musculi intercostales, des Diaphragmas und der inspiratorischen Atemmuskulatur und verbessern das Ventilationsvolumen der Lunge (Wang Dawei 2020).

Beim Taijiquan werden durch Lösen und Durchgängigmachen der Leitbahnen, Dehnen des Brustkorbs und Fördern der Zirkulation von Qi und Xue das Training des Lungenbereichs und die Regeneration der Lungenfunktion unterstützt (Zhang Hao 2019). Andere traditionelle gesundheitserhaltende Übungen wie die "Sechs Laute“ (auch „Lehrformel der Sechs Zeichen“, Liuzi jue) (Hao Shijie 2018) und die „Schildkröten-Übungen“ (He Zirong 2015) haben auf die Wiederherstellung der Lungenfunktion ebenfalls eine gewisse stützende Wirkung. Sie sollten je nach den konkreten klimatischen und geographischen Gegebenheiten und dem Zustand des Patienten vernünftig ausgewählt werden.

\section{$5 \quad$ Klinische Studien}

Seit dem Ausbruch von Covid-19 wurden in ganz China zahlreiche klinische Studien durchgeführt. Diese Studien spiegeln die vielfältigen Behandlungsmöglichkeiten der Chinesischen Medizin wider, und durch sie wurden für die Ausarbeitung der nationalen Pläne und die Bekämpfung der Epidemie mit Chinesischer Medizin wertvolle Erfahrungen gesammelt und klinische Daten geliefert.

Ein Forschungsteam um Zhang Boli hat gezeigt, dass durch die Verwendung des „Infektionen klärenden Granulats mit Lonicerae flos" (Jinhua qinggan keli) ${ }^{9}$ bei der Behandlung von 82 Patienten nach 5 Tagen die klinischen Symptome Fieber, Husten, Kraftlosigkeit und Sputum bei Patienten mit Covid-19 der leichten Form signifikant reduziert und psychische Angstzustände gelindert werden konnten (Duan Can 2020). Yang Zhongjie et al. haben 13 Patienten mit Covid-19 der Form

9 Die Ingredienzen des "Infektionen klärenden Granulats mit Lonicerae flos" (Jinhua qinggan keli 金花清感颗粒) sind Lonicerae flos (Jinyinhua), Gypsum fibrosum (Shigao), Ephedrae herba (Mahuang), Armeniacae semen amarum (Kuxingren), Scutellariae radix (Huangqin), Forsythiae fructus (Lianqiao), Fritillariae thunbergii bulbus (Zhebeimu), Anemarrhenae rhizoma (Zhimu), Arctii fructus (Niubangzi), Artemisiae annuae herba (Qinghao), Menthae herba (Bohe) und Glycyrrhizae radix (Gancao).
Positive Wirkung des "Spiels der Fünf Tiere“

Signifikanter Rückgang der Hauptsymptome 
Neuer Ansatz für die Behandlung der schweren Form
Einstauungen von humor algidus („Kälte-Feuchtigkeit“, hanshi) im o. pulmonalis (Fk „Lunge“, fei) behandelt und festgestellt, dass die „Mixtur mit Astragalus, Cinnamomum und Poria" (Qiguilin heji) ${ }^{10}$ bei den Patienten Symptome wie Fieber, Husten, Kraftlosigkeit, Diarrhoe und Übelkeit deutlich bessern kann. Chen Ling et al. (Chen Ling 2020) haben 131 Covid-19-infizierte Patienten (zu 95,4 \% übliche Form) mit dem „Gift auflösenden Dekokt des süßen Taus“ (Ganlu xiaodu tang) ${ }^{11}$ in Kombination mit chinesischen und westlichen Arzneimitteln erfolgreich behandelt und festgestellt, dass diese Rezeptur am besten mit der Fertigrezeptur "Epidemisches klärendes (Granulat) mit Forsythiae fructus und Lonicerae flos" (Lianhua qingwen), der Fertigrezeptur „Wirkkräftiger Tau mit Eriobotryae folium“ (Qiangli pipa tang) sowie ventus („Wind“, feng) lösenden und Toxisches herauslösenden Arzneimitteln zusammenwirkt.

Xie Yanfeng et al. (Xie Yanfeng 2020) berichten über die erfolgreiche klinische Behandlung von 8 Patienten mit Covid-19 der schweren Form durch Einsatz des "Regeln brechenden Dekoktes, das dem Fk Herz zu Hilfe kommt" (Poge jiuxin tang) und des „Kleinen Dekoktes des grünen Drachen“ (Xiao qinglong tang) ${ }^{12}$, womit sie einen neuen Ansatz für die Behandlung von Covid-19 der schweren Form mit Chinesischer Medizin lieferten. Das „Regeln brechende Dekokt, das dem Fk Herz zu Hilfe kommt" (Poge jiuxin tang) ist auf Grundlage der Erfahrungen

10 Die „Mixtur mit Astragalus, Cinnamomum und Poria” (Qiguilin heji 芪桂苓合剂) basiert hauptsächlich auf dem „Cinnamomum-Dekokt ohne Paeonia“" (Guizhi qu shaoyao tang, s. Scheid 2009, S. 18), dem „Den Fk Magen (o. stomachi) befriedenden Pulver" (Pingwei san, s. Hempen 2006, S. 194), dem "Pulver gegen Wind aus Jade" (Yu pingfeng san, s. Hempen 2006, S. 342) und dem "Dekokt mit Platycodi radix und Glycyrrhhizae radix" (Jiegeng gancao tang, bestehend aus Platycodi radix [Jiegeng], Glycyrrhizae radix [Gancao], Pinelliae rhizoma [Banxia], Inulae flos [Xuanfuhua], Arecae pericarpium [Dafupi], Aurantii fructus [Zhike], Poria rubra [Chifuling], Paeoniae rubrae radix/Paeoniae lactiflorae radix [Shaoyao] und Peucedani radix [Qianhu]).

11 Die „Gift auflösende Pille des süßen Taus” (Ganlu xiaodu dan 甘露消毒丹, s. Hempen 2006, S. 226) ist eine humor („Feuchtigkeit", shi) beseitigende Rezeptur; sie setzt sich zusammen aus $45 \mathrm{~g}$ Talcum (Huashi), $30 \mathrm{~g}$ Scutellariae radix (Huangqin), $30 \mathrm{~g}$ Artemisiae scopariae herba (Yinchenhao), $18 \mathrm{~g}$ Acori rhizoma (Shichangpu), $15 \mathrm{~g}$ Fritillariae cirrhosae bulbus (Chuanbeimu), $15 \mathrm{~g}$ Akebiae caulis (Mutong), $12 \mathrm{~g}$ Agastachis herba (Huoxiang), $12 \mathrm{~g}$ Forsythiae fructus (Lianqiao), $12 \mathrm{~g} \mathrm{Amomi} \mathrm{fructus} \mathrm{rotundus} \mathrm{(Baidoukou),} 12 \mathrm{~g}$ Menthae herba (Bohe) und $12 \mathrm{~g}$ Belamcandae rhizoma (Shegan). Sie wurde in der Qing-Zeit vom Arzt Ye Tianshi entwickelt und ist die wichtigste Rezeptur für die Behandlung von epidemischen Krankheiten aufgrund humorWärme („Feuchtigkeit-Wärme”, shiwen). Die Rezeptur hat eine Toxisches herauslösende, Trübes umwandelnde, calor ("Hitze", re) kühlende und humor ("Feuchtigkeit", shi) ausleitende Wirkung und hat bei Krankheitsbildern, bei denen sich Heteropathien (Schrägläufigkeiten, xie) im Bereich des Qi befinden und bei denen humor („Feuchtigkeit”, shi) und calor („Hitze”, re) gleich stark ausgeprägt sind, eine deutliche Therapiewirkung.

12 „Regeln brechendes Dekokt, das dem Fk Herz zu Hilfe kommt“ (Poge jiuxin tang 破格救心汤) und „Kleines Dekokt des grünen Drachen" (Xiao qinglong tang 小青龙汤): 75 g Aconiti radix lateralis praeparata (Fuzi, zuerst 2 Stunden abkochen), $30 \mathrm{~g}$ Zingiberis rhizoma (Ganjiang), $60 \mathrm{~g}$ in einer Flüssigkeit geröstete Glycyrrhizae radix (Gancao), $30 \mathrm{~g}$ unpräpariertes Mastodi fossilium ossis (Longgu, zuerst 2 Stunden abkochen), $30 \mathrm{~g}$ unpräpariertes Ostreae concha (Muli, zuerst 2 Stunden abkochen), $30 \mathrm{~g}$ Magnetitum (Cishi, zuerst 2 Stunden abkochen), $30 \mathrm{~g}$ Ginseng radix rubra (Hongshen, separat erhitzen), 0,3 g Moschus (Shexiang, Kategorie II im Washingtoner Artenschutzübereinkommen - Handel reglementiert; auf 3 Portionen verteilt einnehmen), $45 \mathrm{~g}$ Atractylodis macrocephalae rhizoma (Baizhu), $45 \mathrm{~g}$ Poria (Fuling), $15 \mathrm{~g}$ Ephedrae herba (Mahuang), $20 \mathrm{~g}$ Armeniacae semen (Xingren), $30 \mathrm{~g}$ Asari radix (Xixin), $45 \mathrm{~g}$ Cinnamomi cassiae ramulus (Guizhi). $45 \mathrm{~g}$ Paeoniae radix rubra (Chishaoyao), $20 \mathrm{~g}$ Schisandrae fructus (Wuweizi), $45 \mathrm{~g}$ unpräparierte Pinelliae rhizoma (Shengbanxia, nur kurzfristig und in schweren Fällen einzusetzen), $30 \mathrm{~g}$ Zingiberis rhizoma recens (Shengjiang). Modifikationen: (1) Bei depletio qi (energetische Schwäche des Qi, qixu) mit Kraftlosigkeit zusätzlich $120 \mathrm{~g}$ Astragali radix (Huangqi), 30 g Cimicifugae rhizoma (Shengma); (2) bei Fieber zusätzlich $120 \mathrm{~g}$ unpräpariertes Gypsum fibrosum (Shigao, in einem Tuch abkochen); (3) bei deutlichen Druckgefühlen im Thorax zusätzlich $30 \mathrm{~g}$ Trichosanthis fructus (Gualou), $30 \mathrm{~g}$ Allii macrostemonis bulbus (Xiebai, halbe Stunde in klarem Branntwein eingeweicht); (4) bei Thoraxerguss zusätzlich $30 \mathrm{~g}$ Lepidii/ Descurainiae semen (Tinglizi), 12 Stück Jujubae fructus (Dazao); (5) bei einem dicken, klebrigen Zungenbelag zusätzlich $30 \mathrm{~g}$ Arecae semen (Binglang), $30 \mathrm{~g}$ Tsaoko fructus (Caoguo). 
des Altarztes Li Ke mit der klinischen Notfallbehandlung kritischer Erkrankungen entstanden; es basiert auf dem „Dekokt mit Ginseng, Aconitum, Mastodi fossilium ossis und Ostreae concha zum Beheben von Kontravektionen“ (Shenfu longmu jiuni tang), das in der "Abhandlung über schädigende Kälte (algor laedens)“ (Shanghan lun, von Zhang Zhongjing, aus dem 2. Jh.) unter den „Dem Dekokt gegen die vier Kontravektionen" (Sini tang) ähnlichen Rezepturen gelistet wird, und auf dem von Zhang Xichun entwickelten „Dekokt, das zurückkehren lässt“ (Laifu tang), wobei Aconiti radix lateralis praeparata (Fuzi) und Corni fructus (Shanzhuyu) hochdosiert verwendet werden und Ginseng radix (Renshen) zugegeben wird (Li Ke 2002).

\section{$6 \quad$ Resümee}

Bisher durchgeführte Studien geben Hinweise, dass sich bei Covid-19 mit Chinesischer Medizin die Symptome lindern, die Weiterentwicklung von der leichten und üblichen Form zur schweren Form reduzieren, die Genesungsrate steigern, die Mortalität senken und die Rekonvaleszenz beschleunigen lassen. Zur Therapie und zur Prävention wird hauptsächlich Phytotherapie eingesetzt, Akupunktur und Moxibustion können unterstützend genutzt werden. In der Rekonvaleszenz können zudem auch chinesische Bewegungstherapien zum Einsatz kommen, um die Genesung zu beschleunigen. Weitere Studien wären wünschenswert.

\section{Literatur}

Chen Ling 陈灵, Cheng Zhiqiang 程志强, Liu Fang 柳芳, Xia Yang 夏杨, Chen Yonggang 陈永刚. "Ganlu xiaodu tang zhiliao 131 li xinxing guanzhuang bingdu feiyan bingli fenxi" 甘露消毒汤 治疗 131例新型冠状病毒肺炎病例分析 (Analyse der Behandlung von 131 Covid-19-Patienten mit dem „Gift auflösenden Dekokt des süßen Taus" (Ganlu xiaodu tang)). China Journal of Chinese Materia Medica 中国中药杂志. 2020,45(10):2232-2238.

Chen Peiyi 陈佩仪. Zhongyi hulixue jichu 中医护理学基础 (Grundlagen der Pflege in der Chinesischen Medizin). Beijing: Renmin weisheng chubanshe人民卫生出版社 (Volksverlag für Hygiene), 2014:73.

Chen Renshou 陈仁寿, Wang Jiahao 王家豪, Shi Zheng 施铮.,Xinxing guanzhuang bingdu feiyan zhongyiyao zhenliao fangan fenxi yu sikao" 新型冠状病毒肺炎中医药诊疗方案分析与思考 (Analyse der TCM-Diagnose- und -Behandlungspläne für Covid-19 und Gedanken dazu). Jiangsu Journal of Traditional Chinese Medicine 江苏中医药. 2020,52(04):60-64.

Duan Can 段枝, Xia Wenguang 夏文广, Zheng Chanjuan 郑婵娟, Sun Guobing 孙国兵, Li Zhengliang 李正良, Li Qinglin 李庆琳, Li Ping 李平, Zhang Heling 张荷玲, Yang Fengwen 杨丰文, Zhang Boli 张伯礼, Liu Qingquan 刘清泉. „Jinhua qinggan keli zhiliao xinxing guanzhuang bingdu ganran feiyan de linchuang guancha“金花清感颗粒治疗新型冠状病毒感染肺炎的临 床观察 (Klinische Untersuchung der Behandlung von Covid-19 mit dem „Infektionen klärenden Granulat mit Lonicerae flos" (Jinhua qinggan keli)). Journal of Traditional Chinese Medicine 中医杂志. 2020, http://kns.cnki.net/kcms/detail/11.2166.R.20200323.0853.002.html.

Hao Shijie 郝世杰, Li Linlin 李琳琳, Bi Hongyan 毕鸿雁, Liu Xihua 刘西花, Sun Chao 孙超, Yang Yuru 杨玉如.,Liuzijue lianhe xijiji xunlian dui naocuzhong piantan huanzhe feigongneng de yingxiang”六字诀联合吸气肌训练对脑卒中偏癖患者肺功能的影响 (Der Einfluss der "Lehrformel der Sechs Zeichen" und des Trainings der inspiratorischen Atemmuskulatur auf die Lungenfunktion von Patienten mit Hemiplegie nach Apoplex). Chinese Journal of Rehabilitation 中国康 复. 2018,33(02): 107-110.

He Zirong 何姿蓉. Wudang guixinggong dui manxing zusexing feijibing huanzhe huxi gongneng $j i$ shengcun zhiliang yingxiang de yanjiu 武当”龟形功”对慢性阻塞性肺疾病患者呼吸功能及生 存质量影响的研究 (Untersuchung des Einflusses der Wudang-„SChildkröten-Übungen“ auf die Atemfunktion und die Lebensqualität von COPD-Patienten). 成都中医药大学 (Chengdu University of Traditional Chinese Medicine), 2015.

Hempen C-H, Fischer S, Hummelsberger J, Koch A, Leonhardy H, Nögel R, Thede C, Wullinger M: Leitfaden Chinesische Rezepturen. München: Elsevier, 2006.
Die wichtigsten Effekte der

Chinesischen Medizin bei Covid-19 
Huang Xianbao 黄仙保, Xie Dingyi 谢丁一, Qiu Qi 邱祺, Shen Yang 沈洋, Jiao Lin 焦琳, Li Qiaolin 李 巧林, Chen Rixin 陈日新.,,Reminjiu zhiliao xinxing guanzhuang bingdu feiyan linchuang guancha”热敏炎治疗新型冠状病毒肺炎临床观察 (Klinische Beobachtung der Behandlung von Covid-19 mit schwebender Moxibustion). Chinese Acupuncture \& Moxibustion 中国针炎. https://doi.org/10.13703/j.0255-2930.20200312-k0003.

Huang Xiaoqing 黄晓青, Nie Linghui 聂玲辉, Li Feimeng 黎飞猛, Wang Chun 王春, Chen Gao 陈高, Cai Keke 蔡可可,Yu Chaoyue 于超月，Li Cheng 黎程.„35 li xinxing guanzhuang bingdu feiyan huanzhe zhongyi linchuang tezheng fenxi“ 35例新型冠状病毒肺炎患者中医临床特征分析 (Analyse der klinischen Charakteristika von 35 Patienten mit Covid-19) nach der chinesischen Medizin). Journal of Emergency in Traditional Chinese Medicine 中国中医急症. 2020,29(03):381-383,398.

Li Ke 李可. Li Ke laozhongyi jiwei zhongzheng yinanbing jingyan zhuanji 李可老中医急危重症疑难 病经验专辑 (Sonderausgabe: Erfahrungen des Altarztes Li Ke mit kritischen schwer behandelbaren Erkrankungen). Taiyuan: Shanxi kexue jishu chubanshe山西科学技术出版(Shanxi Science Technology Publishing House), 2002:4-93.

Li Yuefeng 李越峰, Zhang Yugui 张育贵, Niu Jiangtao 牛江涛, Si Xinlei 司昕蕾, Yan Xingke 严兴科. "Zhongyiyao fangzhi xinxing guanzhuang bingdu feiyan suozhi chuangshang hou yingji zhang'ai yongyao tanxi“ 中医药防治新型冠状病毒肺炎所致创伤后应激障碍用药探析 (Untersuchung und Analyse der zur Prävention und Therapie von durch Covid-19 ausgelöster Posttraumatischen Belastungsstörung eingesetzten chinesischen Arzneimittel). Chinese Traditional and Herbal Drugs 中草药. 2020,51(05):1130-1138.

Liu Bing 刘兵, Wang Hua 王华, Zhou Zhongyu 周仲瑜, Chang Xiaorong 常小荣, Zhang Wei 章薇, Liu Baoyan 刘保延.,Zhenjiu fangzhi xinxing guanzhuang bingdu feiyan lilun yu linzheng silu tanxi“ 针炎防治新型冠状病毒肺炎理论与临证思路探析 (Theorie der Prävention und Therapie von Covid-19 mit Akupunktur und Moxibustion und Gedanken, Untersuchung und Analyse der Symptomkonfigurationen). Chinese Acupuncture \& Moxibustion 中国针炎. 2020, https:// doi.org/10.13703/j.0255-2930.20200305-k0004

Luo L, Jiang J, Wang C, Fitzgerald M, Hu W, Zhou Y, Zhang H, Chen S. Analysis on herbal medicines utilized for treatment of COVID-19. Acta Pharmaceutica Sinica B, 2020, May 27 (in press).

Pan Xiaoqiong 潘晓琼, Hu Zhen 胡縥., 64 li xinxing guanzhuang bingdu feiyan putongxing huanzhe zhongyi linchuang tezheng fenxi" 64例新型冠状病毒肺炎普通型患者中医临床特征分析 (Analyse der klinischen Charakteristika nach der chinesischen Medizin von 64 Patienten mit Covid-19). Journal of Wenzhou Medical University 温州医科大学学报. 2020,50(03):187-190.

Peng Bo 彭博, Wang Shichang 王世长, Gao Tongtong 高䑣形, Zhang Haili 张海力, Zhang Jing 张婧, Li Jing 李靖., Cong shidu xiefeng lunzhi yanzheng fengbao yinfa de xinxing guanzhuang bingdu feiyan de bingcheng yanjin“从湿毒挟风论治炎症风暴引发的新型冠状病毒肺炎的病程 演进 (Die Behandlung von durch einen Zytokinsturm hervorgerufener Covid-19 ansetzend bei "Feuchtigkeit"-Toxischem mit "Wind"). World Chinese Medicine 世界中医药. 2020,15(03):315-319.

Scheid V, Bensky D, Ellis A, Barolet R: Chinese Herbal Medicine. Formulas \& Strategies (2nd Edition). Seattle: Eastland Press, 2009.

Shi Suofang 史锁芳, Liu Qingquan 刘清泉.,Cong jiangxia fangcang zhongyi moshi tantao zhongyiyao zai xinxing guanzhuang bingdu feiyan zhiliaozhong de jiazhi“从“江夏方舱中医模式”探讨 中医药在新型冠状病毒肺炎治疗中的价值 (Die Bedeutung der TCM bei der Behandlung von Covid-19 auf Grundlage des TCM-Modells im Kabinenkrankenhaus Jiangxia). Jiangsu Journal of Traditional Chinese Medicine 江苏中医药. 2020, https://doi.org/10.19844/j.cnki.1672397X.2020.00.008.

Shi Xuemin 石学敏, Tong Xiaolin 全小林, Sun Guojie 孙国杰, Liu Baoyan 刘保延, Wang Hua 王华, Yu Xiaochun 喻晓春, Wu Huangan 吴焕涂, Gao Shuzhong 高树中, Wang Linpeng 王麟鹏, Fang Jianqiao 方剑乔, Yu Shuguang 余曙光, Liang Fanrong 梁繁荣, Ji Laixi 冀来喜, Jing Xianghong 景向红, Zhou Zhongyu 周仲瑜, Ma Jun 马骏, Chang Xiaorong 常小荣, Zhang Wei 章薇, Yang Jun 杨骏, Chen Rixin 陈日新, Zhao Jiping 赵吉平, Zhao Hong 赵宏, Zhao Baixiao 赵百孝, Wang Fuchun 王富春, Liang Fengxia 梁风霞, Li Xiaodong 李晓东, Yang Yi 杨毅, Liu Weihong 刘炜宏， Wen Biling 文碧玲. „Xinxing guanzhuang bingdu feiyan zhenjiu ganyu de zhidao yijian (di er ban)“ 新型冠状病毒肺炎针炎干预的指导意见(第二版) (Anleitung zur Akupunktur- und Moxibustion-Intervention bei Covid-19, 2. Auflage). Chinese Acupuncture \& Moxibustion 中国针炎. 2020,40(05):462-463.

State Council Information Office of the People's Republic of China 国务院新闻办公室网站.,Zhongyiyao zongyouxiaolü da 90\% yishang" 中医药总有效率达 $90 \%$ 以上 (Die Gesamtwirkungsrate der Behandlung mit chinesischer Medizin liegt bei über $90 \%$ ). http://www.scio.gov.cn/xwfbh/ xwbfbh/wqfbh/42311/42768/zy42772/Document/1675815/ 1675815.htm, Mar. 23, 2020/Jun. 13, 2020.

Wang Dawei 王大伟, Tian Lingling 田玲玲, Zhao Yanjie 赵延杰, Song Xiedao 宋修道, Li Wei 李炜. „Zhongyi bianzheng lianhe wuqinxi zhiliao manxing zusexing feijibing 60 li linchuang guancha 
中医辨证联合五禽戏治疗慢性阻塞性肺疾病60例临床观察 (Klinische Beobachtung der Behandlung von 60 COPD-Patienten durch Bestimmung der Symptomkonfiguration nach der Chinesischen Medizin in Kombination mit dem „Spiel der FünfTiere"). Chinese Journal of Convalescent Medicine 中国疗养医学. 2020 (2), 123-125.

Wang Raoqiong 王饶琼, Yang Sijin 杨思进, Xie Chunguang 谢春光, Shen Qilin 沈其霖, Li Minqing 李敏清, Lei Xiao 雷香, Li Jike 李继科, Huang Mei 黄梅., Qingfei paidu tang zhiliao xinxing guanzhuang bingdu feiyan de linchuang liaoxiao guancha“清肺排毒汤治疗新型冠状病毒肺炎 的临床疗效观察 (Klinische Beobachtung des „Den o. pulmonalis kühlenden und Toxisches ausscheidenden Dekoktes" (Qingfei paidu tang) bei der Behandlung von durch das neuartige Coronavirus hervorgerufener Pneumonie). Pharmakologie und Klinik chinesischer Arzneimittel 中药药理与临床 2020;36(1), S. 13-8.

Wang Wei 王薇, Wang Yuwei 王玉伟, Ma Shuang 马爽, Li Ruifeng 李瑞锋.,Gesheng zhongyi zhiliao celüe ji zhongyi zhiliao canyulü yu xinxing guanzhuang bingdu feiyan zhiyu xiaoguo chutan“ 各省中医治疗策略及中医治疗参与率与新型冠状病毒肺炎治愈效果初探 (Strategie und Beteiligungsrate der TCM-Behandlung in den einzelnen Provinzen und erste Therapieergebnisse bei Covid-19). World Chinese Medicine 世界中医药. 2020, http://kns.cnki.net/kcms/detail/11.55 29.R.20200306.1345.004.html.

Xia Wenguang 夏文广，Hua Qiang 华强,Wang Gang 王刚, Guo Tiecheng 郭铁成, Zhu Shanshan 朱 珊珊, Zhao Yan 赵焰, Tian Jun 田俊, Zheng Chanjuan 郑婵娟, Zhang Yangpu 张阳普, Zhang Wei 张伟, Tang Qiang 唐强, Jin Rongjiang 金荣疆, Feng Xiaodong 冯晓东, Huang Xiaolin 黄晓 琳, Zhang Boli张伯礼. „Xinxing guanzhuang bingdu feiyan zhongxiyi jiehe kangfu zhenliao guifan“新型冠状病毒肺炎中西医结合康复诊疗规范 (Standards für Diagnose und Behandlung in der Rekonvaleszenz von Covid-19 mit der Kombination von Chinesischer und westlicher Medizin). Rehabilitation Medicine 康复学报. 2020, 30(2):94-101

Xie Yanfeng 谢炎烽, Ruan Yongdui 阮永队, Liu Xiaoru 刘晓茹, Zhuang Xiaomin 庄晓敏.,Zhongyiyao zhiliao 8 li zhongxing xinxing guanzhuang bingdu feiyan jingyan tantao“ 中医药治疗 8 例 重型新型冠状病毒肺炎经验探讨 (Die Erfahrungen mit der Behandlung von 8 Patienten mit Covid-19 der schweren Form mit chinesischen Arzneimitteln). Journal of Practical Traditional Chinese Internal Medicine 实用中医内科杂志. 2020, https://doi.org/10.13729/j.issn.1671-7813. Z20200188

Xu Erping 许二平, Xu Jiangyan 徐江雁, Xu Liran 徐立然, Wei Zheng 魏征, Meng Pengfei 孟鹏飞, Song Xiyuan 宋夕元, Ding Xue 丁雪, Cai Yongmin 蔡永敏, Zhang Haiyan 张海燕., Xinxing guanzhuang bingdu feiyan changjian bingzheng jingfang bianzhi gangyao“新型冠状病毒肺炎 常见病症经方辨治纲要 (Überblick über die Bestimmung der Symptomkonfiguration und die Behandlung mit klassischen Rezepturen bei häufigen Symptomen bei Covid-19). Acta Chinese Medicine 中医学报. 2020,35(04):679-681.

Yang Zhongjie 杨忠杰, Ye Lin 叶琳, Chen Lang 陈朗, Miao Mingsan 苗明三, Li Yaojun 李耀军.,QQiguiling heji zhiliao xinxing guanzhuang bingdu feiyan“花桂苓合剂治疗新型冠状病毒肺炎 (Die Behandlung von Covid-19 mit der "Mixtur mit Astragalus, Cinnamomum und Poria“ (Qiguilin heji)). Acta Chinese Medicine 中医学报. 2020(06):1151-1153+1176[2020-07-08]. https://doi. org/10.16368/j.issn.1674-8999.2020.06.257.

Zhang H. Bamai Qigong. Aarhus, Heyttu Academic Publishing, 2020:13-16.

Zhang Hao 张妤, Liu Dunhuang 刘敦煌.„Ershisi shi taijiqun jiehe huxi kangfu xunlian dui manxing zusexing feijibing huanzhe feigongneng ji shenghuo zhiliang de yingxiang"二十四式太极拳 结合呼吸康复训练对慢性阻塞性肺疾病患者肺功能及生活质量的影响 (Einfluss der 24 Formen des Taijiquan in Kombination mit Atemübungen für die Rekonvaleszenz auf die Lungenfunktion und die Lebensqualität von COPD-Patienten). Journal of Practical Traditional Chinese Medicine 实用中医药杂志. 2019,35(3):350-351.

Zhang Yuan 张圆, Wang Yuguang 王玉光, Cheng Haiying 程海英.„Xinxing guanzhuang bingdu feiyan chuyuan huanzhe zhongyi kangfu zhiliao silu yu fangfa“新型冠状病毒肺炎出院患者中 医康复治疗思路与方法 (Wege und Methoden der Behandlung mit Chinesischer Medizin in der Rekonvaleszenz von Covid-19 bei aus dem Krankenhaus entlassenen Patienten). Beijing Journal of Traditional Chinese Medicine 北京中医药. 2020,39(03):227-229.

Zhou Jing 周静, Chen Jingjing 陈晶晶, Zhang Nianzhi 张念志, Zhu Xiaoxiao 朱笑笑, Li Jing 李晶, Wang Shiqing 汪诗清, Li Yundong 李允栋, Han Mingxiang 韩明向, Chen Wei 陈炜, Dong Mei 董梅, Liu Ling 刘玲, Hou Haobin 侯浩涁, Ding Zhen 丁震, Liu Xinkuang 刘新矿, Yao Li 姚莉. "Anhui sheng 144 li xinxing guanzhuang bingdu feiyan huanzhe zhongyi zhenghou tezheng" 安徽省 144例新型冠状病毒肺炎患者中医证候特征 (Charakteristika der Symptomkonfigurationen nach der chinesischen Medizin von 144 Covid-19-Patienten in der Provinz Anhui). Chinese Journal of Experimental Traditional Medical Formulas 中国实验方剂学杂志. 2020,26(12):28-34. 


\section{Zum Autor}

Dr. Zhang Hui, Ph.D., absolvierte sein Studium an der TCM-Universität Chengdu, es folgten drei Jahre Forschung mit zahlreichen Publikationen; 2011 erwarb er das MA-Diplom mit dem Schwerpunkt TCM-Onkologie, 2017 seinen Ph.D.

Nach einer Assistentenzeit am Sichuan Provincial Hospital for Integrative Chinese and Western Medicine wurde er Dozent an der TCM-Universität Chengdu. Dort unterrichtete er in der Theorie der TCM und behandelte Patienten an der TCM-Klinik Xinglin. Seit 2017 arbeitet und lehrt Dr. Zhang an der AkupunkturAkademiet in Aarhus, Dänemark.

Dr. Zhang hat bei verschiedenen Altärzten besondere Formen der Akupunktur erlernt. Ein Hauptinteresse von Dr. Zhang gilt dem Studium der Klassiker der Chinesischen Medizin.

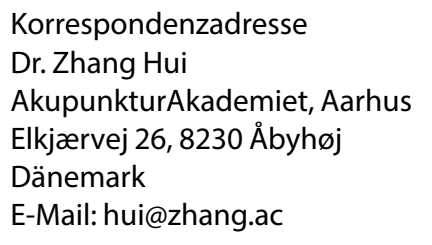

Einhaltung ethischer Richtlinien

Interessenkonflikt: Hui Zhang, Lu Luo, Zhi Qing Liu und Hui Xie geben an, dass kein Interessenkonflikt besteht. Dieser Beitrag beinhaltet keine Studien an Menschen oder Tieren. 